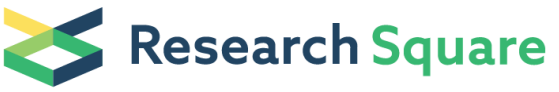

Preprints are preliminary reports that have not undergone peer review.

They should not be considered conclusive, used to inform clinical practice, or referenced by the media as validated information.

\section{Long Non-Coding RNA CRNDE is Involved in Resistance to EGFR Tyrosine Kinase Inhibitor in EGFR-Mutant Lung Cancer via elF4A3/MUC1/EGFR Signaling}

\section{Satoshi Takahashi}

Division of Pulmonary Medicine and Oncology, Graduate School of Medicine, Nippon Medical School

\section{Rintaro Noro}

Division of Pulmonary Medicine and Oncology, Graduate School of Medicine, Nippon Medical School Masahiro Seike ( $\nabla$ mseike@nms.ac.jp)

Division of Pulmonary Medicine and Oncology, Graduate School of Medicine, Nippon Medical School Chao Zeng

Department of Electrical Engineering and Bioscience, Faculty of Science and Engineering, Waseda University

\section{Masaru Matsumoto}

Division of Pulmonary Medicine and Oncology, Graduate School of Medicine, Nippon Medical School

\section{Akiko Yoshikawa}

Division of Pulmonary Medicine and Oncology, Graduate School of Medicine, Nippon Medical School

\section{Shinji Nakamichi}

Division of Pulmonary Medicine and Oncology, Graduate School of Medicine, Nippon Medical School

\section{Teppei Sugano}

Division of Pulmonary Medicine and Oncology, Graduate School of Medicine, Nippon Medical School

\section{Mariko Hirao}

Division of Pulmonary Medicine and Oncology, Graduate School of Medicine, Nippon Medical School

\section{Kuniko Matsuda}

Division of Pulmonary Medicine and Oncology, Graduate School of Medicine, Nippon Medical School

\section{Michiaki Hamada}

Department of Electrical Engineering and Bioscience, Faculty of Science and Engineering, Waseda University

\section{Akihiko Gemma}

Division of Pulmonary Medicine and Oncology, Graduate School of Medicine, Nippon Medical School

\section{Research Article}


Keywords: EGFR-TKI, drug resistance, CRNDE, elF4A3

Posted Date: January 13th, 2021

DOI: https://doi.org/10.21203/rs.3.rs-137571/v1

License: (c) (1) This work is licensed under a Creative Commons Attribution 4.0 International License. Read Full License 


\section{Abstract}

Introduction: Acquired resistance to epidermal growth factor receptor-tyrosine kinase inhibitors (EGFRTKIs) is an intractable challenge for many clinical oncologists. The mechanisms of resistance to EGFRTKIs are complex. Long non-coding RNAs (IncRNAs) may play an important role in cancer development and metastasis. However, the biological process between IncRNAs and drug resistance to EGFR-mutated lung cancer remains largely unknown.

Methods: Osimertinib- and afatinib-resistant EGFR-mutated lung cancer cells were established using a stepwise method. Microarray analysis of non-coding and coding RNAs was performed using parental and resistant EGFR-mutant non-small cell lung cancer (NSCLC) cells and evaluated by bioinformatics analysis through medical-industrial collaboration.

Results: Colorectal neoplasia differentially expressed (CRNDE) and DiGeorge syndrome critical region gene 5 (DGCR5) IncRNAs were highly expressed in EGFR-TKI-resistant cells by microarray analysis. RNAprotein binding analysis by medical-industrial collaboration revealed eukaryotic translation initiation factor 4A3 (eIF4A3) bound overlappingly to CRNDE and DGCR5. The CRNDE downregulates the expression of elF4A3, mucin 1 (MUC1), and phospho-EGFR. Inhibition of CRNDE activated the elF4A3/MUC1/EGFR signaling pathway and apoptotic activity, and restored sensitivity to EGFR-TKIs.

Conclusions: The results showed that IncRNA CRNDE is associated with the development of resistance to EGFR-TKIs in EGFR-mutant NSCLC cells. CRNDE may be a novel therapeutic target to conquer EGFRmutant NSCLC.

\section{Introduction}

Epidermal growth factor receptor-tyrosine kinase inhibitors (EGFR-TKIs) have shown marked efficacy in non-small cell lung cancer (NSCLC) patients with EGFR mutations ${ }^{1-6}$. Osimertinib (a third-generation EGFR-TKI) showed significant efficacy in NSCLC patients with and without the T790M mutation in exon 20 , which was previously considered a second-hit mutation 7,8 . Acquired mechanisms of resistance after treatment with osimertinib, including C797S mutation, activation of bypass pathway such as MET amplification, BRAF mutation, and transformation of small cell lung cancer have been previously reported ${ }^{9}$. However, therapeutic strategies for overcoming resistance to EGFR-TKIs have not yet been established.

In addition to mutations and aberrant expression of protein-encoding genes, non-coding RNAs (including microRNAs) appear to play a major role in cancer. We have previously reported that the miR-21, miR134/487b/655, and miR-200 families can be used as therapeutic targets associated with EGFR-TKI treatment ${ }^{10-12}$. Long non-coding RNAs (IncRNAs), defined as transcripts longer than 200 nucleotides, do not have the ability to encode proteins. LncRNAs play an important role in the development of tumors in a variety of human cancers, including lung cancer. Recently, IncRNAs have been shown to regulate gene 
expression and participate in multiple biological processes, such as cell proliferation, migration, metastasis, apoptosis, and drug resistance ${ }^{13,14}$. Moreover, they exhibit tumor suppressor and carcinogenic functions, and have been identified as a promising novel biomarker and therapeutic target for lung cancer. However, there is limited knowledge regarding their roles in biological processes and drug resistance in lung cancers with EGFR mutations.

In this study, we used comprehensive IncRNA expression profiles and medical-industrial bioinformatics techniques to identify IncRNAs associated with the mechanism of acquired resistance to EGFR-TKIs in EGFR-mutant NSCLC cells.

\section{Results}

\section{Effect of afatinib and osimertinib on lung adenocarcinoma cells and establishment of resistant cell lines}

We evaluated the anti-tumor of afatinib and osimertinib in four EGFR-mutant lung adenocarcinoma cell lines (i.e., PC-9, HCC827, H1975, and H1650) by the 3-(4,5-dimethylthiazol-2-yl)-5-(3carboxymethoxyphenyl)-2-(4-sulfophenyl)-2H-tetrazolium, inner salt (MTS) assay. Whenever PC-9 and HCC827 cells were sensitive to afatinib and osimertinib, $\left(\mathrm{IC}_{50}\right.$ : $\mathrm{PC}-9$ afatinib: $<0.001 \mu \mathrm{M}$, osimertinib: $0.004 \pm 0.0008 \mu \mathrm{M}$ and HCC827 afatinib: $<0.001 \mu \mathrm{M}$, osimertinib: $<0.001 \mu \mathrm{M}), \mathrm{H} 1975$ and $\mathrm{H} 1650$ cells were resistant to these agents $\left(\mathrm{IC}_{50}\right.$ : $\mathrm{H} 1975$ afatinib: $3.3 \pm 1.9 \mu \mathrm{M}$, osimertinib: $2.8 \pm 1.2 \mu \mathrm{M}$ and $\mathrm{H} 1650$ afatinib: $2.3 \pm 0.33 \mu \mathrm{M}$, osimertinib: $2.1 \pm 0.27 \mu \mathrm{M})$ (Table 1). 
Table 1

Characteristics and $\mathrm{IC}_{50}$ of parental and established EGFR-TKI-resistant NSCLC cells

\begin{tabular}{|c|c|c|c|c|c|}
\hline & \multicolumn{2}{|l|}{ Afatinib } & \multicolumn{2}{|l|}{ Osimertinib } & \multirow[t]{3}{*}{ Mutation } \\
\hline & $\mathrm{IC}_{50}$ value $(\mu \mathrm{M})$ & P-value & $I_{50}$ value $(\mu \mathrm{M})$ & P-value & \\
\hline & \multicolumn{2}{|l|}{$($ Mean \pm SD $)$} & \multicolumn{2}{|l|}{$($ Mean \pm SD $)$} & \\
\hline PC-9 & \multicolumn{2}{|l|}{$<0.001$} & \multicolumn{2}{|l|}{$0.004 \pm 0.0008$} & A deletion in exon 19 \\
\hline PC-9-AR & $2.3 \pm 0.32$ & $<0.001$ & - & & \\
\hline PC-9-OR & \multicolumn{2}{|l|}{-} & $2.4 \pm 0.10$ & $<0.001$ & \\
\hline HCC827 & \multicolumn{2}{|l|}{$<0.001$} & \multicolumn{2}{|l|}{$<0.001$} & A deletion in exon 19 \\
\hline HCC827-AR & $2.4 \pm 0.84$ & 0.016 & - & & \\
\hline HCC827-OR & \multicolumn{2}{|l|}{-} & $3.1 \pm 0.58$ & 0.002 & \\
\hline H1975 & \multicolumn{2}{|l|}{$3.3 \pm 1.9$} & \multicolumn{2}{|l|}{$2.8 \pm 1.2$} & L858R/T790M \\
\hline \multirow[t]{2}{*}{ H1650 } & \multirow[t]{2}{*}{$2.3 \pm 0.33$} & & \multirow[t]{2}{*}{$2.1 \pm 0.27$} & & A deletion in exon 19 \\
\hline & & & & & /PTEN null \\
\hline \multicolumn{6}{|c|}{$\begin{array}{l}\text { Abbreviations: AR, afatinib-resistant; EGFR-TKI, epidermal growth factor receptor-tyrosine kinase } \\
\text { inhibitor; IC }{ }_{50} \text {, concentration of drug needed for } 50 \% \text { inhibition of growth; OR, osimertinib-resistant; } \\
\text { NSCLC, non-small cell lung cancer; SD, standard deviation }\end{array}$} \\
\hline
\end{tabular}

We established afatinib-resistant (AR) or osimertinib-resistant (OR) cell lines from the parental lines PC-9 and $\mathrm{HCC} 827$ through a stepwise method ${ }^{12,15}$. After 6 months, we established four drug-resistant cell lines: PC-9-AR, PC-9-OR, HCC827-AR, and HCC827-OR. We confirmed the stability of the cell lines that became resistant for at least 12 months without the addition of any reagents. We also compared the dose-dependence of the parent cell lines and the established resistant cell lines to each drug. The $\mathrm{IC}_{50}$ values of the resistant cell lines were almost 1,000-fold higher than those of the parental cell lines (Fig. 1a and Table 1). These resistant cell lines did not have secondary mutations, such as T790M, C797S, and MET amplification ${ }^{12}$.

We examined the protein expression levels of EGFR signaling pathway molecules in parental cells and EGFR-TKIs-resistant cells by western blotting. We observed decreased protein levels of phospho-EGFR ( $p$ EGFR) in AR and OR cells and increased protein levels of $p$-AKT and p-extracellular signal-regulated kinase ( $p$-ERK) in the three established resistant cells, except for HCC827-AR. These findings suggested the involvement of EGFR signaling-independent mechanisms (Fig. 1b).

\section{Overexpression of colorectal neoplasia differentially expressed (CRNDE) in EGFR-TKI-resistant cell lines}


We next performed non-coding and coding RNA microarray analysis to evaluate the post-transcriptional regulation associated with drug resistance using the parental and EGFR-TKI-resistant cells. In total, we analyzed the expression of 27,246 messenger RNAs (mRNAs) and 25,945 IncRNA (Supplementary Table S1). We also normalized differences in the expression of mRNAs and IncRNAs (Supplementary Fig. S1).

We mapped mRNAs, IncRNAs, and other RNAs based on available bioinformatics tools and databases that are useful in the search for non-coding RNAs and analysis of their structure, conservation, interactions, co-expression, and localization ${ }^{16}$. Candidate IncRNAs and mRNAs were determined by the difference in expression according to a cutoff value. Of note, they exhibited more than two-fold higher expression versus those of resistant and parental cells (Fig. 2a). CRNDE and DiGeorge syndrome critical region gene 5 (DGCR5) IncRNAs showed higher expression levels in resistant cells than in parental cells (Fig. 2a). In addition, these IncRNAs were more highly expressed in intrinsically resistant cells (e.g., H1975 and $\mathrm{H} 1650$ cells) than in sensitive cells (e.g., PC-9 and HCC827 cells) (Fig. 2b). Increased expression of CRNDE and DGCR5 was also observed in established resistant cell lines by quantitative reverse transcription-polymerase chain reaction (qRT-PCR) analysis (CRNDE: PC-9 vs. PC9-AR and PC9-OR, $\mathrm{P}<$ 0.01 and $P=0.07$, respectively; HCC827 vs. HCC827-AR and HCC827-OR, $P=0.10$ and $P=0.11$, respectively; DCGR5: $P C 9$ vs. $P C 9-A$ and $R$ and $P C 9-O R, P=0.31$ and $P=0.23$, respectively; HCC827 vs. HCC827-AR and HCC827-OR, $P=0.59$ and $P=0.73$, respectively) (Figs. 2c, d).

RNA-protein binding data revealed 23 and six proteins correlated with CRNDE and DGCR5, respectively. Eukaryotic translation initiation factor 4A3 (elF4A3) bound overlappingly to CRNDE and DGCR5 (Table 2). CRNDE bound directly to elF4A3, which was the most abundant protein in EGFR-TKI-resistant cells (Fig. 3a). Therefore, we assessed the expression of elF4A3 in EGFR-TKI-resistant cells by qRT-PCR and western blotting. The levels of elF4A3 were decreased in EGFR-TKI-resistant cells compared with the parental cells (Figs. 3b, c). We subsequently assessed the protein expression of elF4A3 downstream signaling molecules in parental and EGFR-TKI-resistant cells. The analysis showed that the levels of mechanistic target of rapamycin kinase (mTOR), programmed cell death 4 (PDCD4), and p-p70-S6 were increased in EGFR-TKI-resistant cells. In contrast, those of mucin 1-C (MUC1-C) and MUC1 were decreased in the resistant cells (Fig. 3d). 
Table 2

RNA-protein binding data

\begin{tabular}{|c|c|c|c|}
\hline CRNDE & & & DGCR5 \\
\hline EIF4A3 & IGF2BP1 & EWSR1 & EIF4A3 \\
\hline FMRP & IGF2BP2 & FUS-mutant & FMRP \\
\hline FUS & IGF2BP3 & TAF15 & FUS \\
\hline LIN28A & TNRC6 & U2AF65 & LIN28A \\
\hline UPF1 & FXR2 & TIA1 & UPF1 \\
\hline DGCR8 & LIN28B & TIAL1 & SFRS1 \\
\hline HuR & LIN28 & hnRNPC & \\
\hline PTB & ZC3H7B & & \\
\hline \multicolumn{4}{|c|}{ 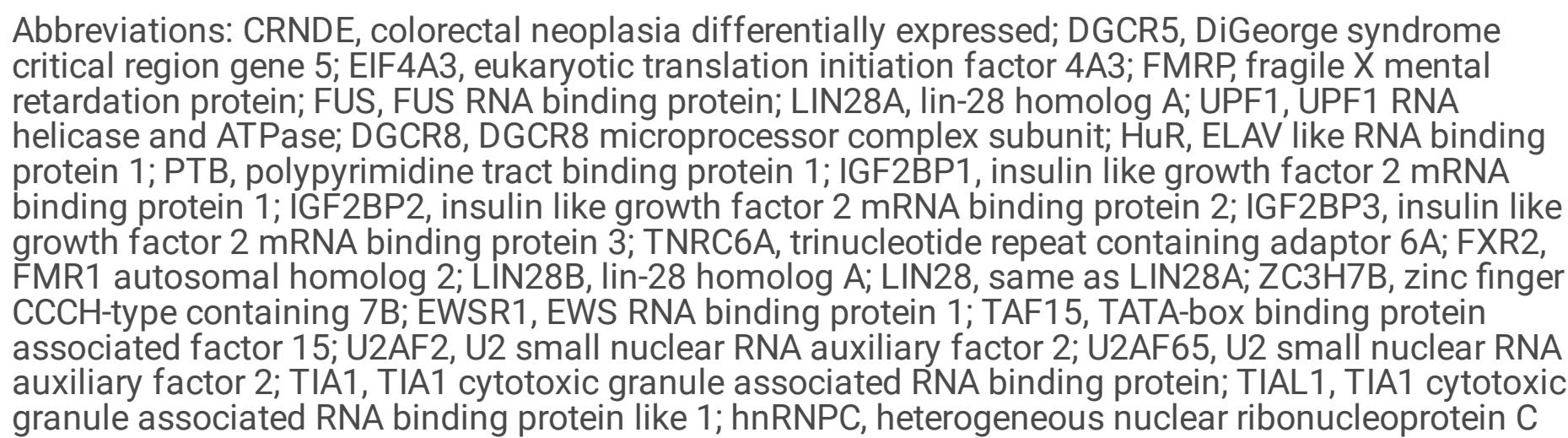 } \\
\hline
\end{tabular}

\section{CRNDE inhibition induced apoptotic activity and overcame the resistance to EGFR-TKIs}

We next assessed the effects of CRNDE on the restoration of elF4A3 expression, apoptotic activity, and sensitivity to EGFR-TKIs using specific small-interfering RNAs (siRNAs). Figure 4a shows that transfection with CRNDE siRNA inhibited the expression of CRNDE in HCC827-OR cells by $90 \%$. Knockdown of CRNDE was achieved by the inhibition of elF4A3 and elF4A3 downstream signaling molecules, such as MUC1-C protein (Fig. 3b). We also observed an increase in p-EGFR expression upon inhibition of CRNDE (Fig. 4b). In contrast, the protein levels of the AKT/mTOR pathway were unchanged, suggesting that CRNDE independently affected the expression levels of elF4A3 protein (Fig. 4b).

We also assessed the apoptotic activity induced by CRNDE inhibition combined with osimertinib treatment. After silencing CRNDE, the protein levels of cleaved poly (ADP-ribose) polymerase (PARP) and cleaved caspase 3 (CASP3) increased (Fig. 4c). Annexin V analysis demonstrated that inhibition of CRNDE also increased the number of apoptotic cells at various doses of osimertinib compared with control siRNA (Fig. 4d). 
Finally, we evaluated whether inhibition of CRNDE restores resistance to EGFR-TKIs in EGFR-mutant NSCLC cells. We measured the response to EGFR-TKIs in HCC827-AR and HCC827-OR cells after treatment with CRNDE siRNA. Knockdown of CRNDE restored resistance to EGFR-TKIs in HCC827-OR and HCC827-AR (Fig. 4e). Therefore, inhibition of CRNDE may activate the elF4A3/MUC1/EGFR signaling pathway and restore sensitivity to EGFR-TKIs (Fig. 5).

\section{Discussion}

Using non-coding and coding RNA microarray analysis, we have identified two IncRNAs, (CRNDE and DGCR5) associated with resistance to EGFR-TKIs in EGFR-mutant NSCLC cells. Through a medicalindustrial collaboration, we successfully identified the target protein of CRNDE and DGCR5, namely elF4A3. It has been reported that IncRNAs function at three levels: transcriptional, post-transcriptional, and epigenetic regulatory ${ }^{14}$. However, the mechanisms by which IncRNAs directly or indirectly promote carcinogenesis, metastasis, and drug resistance have not been elucidated. The interactions of IncRNAs with their coding genes and proteins remain unclear. In this study, we examined these interactions based on the mechanisms associated with resistance to EGFR-TKIs. Direct target discovery of IncRNAs using bioinformatics techniques through a medical-industrial collaboration is innovative and creative.

RNA-protein binding data showed that elF4A3 bound to CRNDE and DGCR5 IncRNAs. Previous studies reported that DGCR5 plays an important role in the development of lung cancer ${ }^{17,18}$. In the present study, despite DGCR5 overexpression, silencing of DGCR5 did not alter the elF4A3/MUC1 signal and p-EGFR, and did not significantly reduce the $\mathrm{IC}_{50}$ value of osimertinib with or without transfection of DGCR5 siRNA (data not shown). Thus, we did not select DGCR5 as a candidate IncRNA.

CRNDE is an oncogenic IncRNA located at an atypical locus-hCG_1815491 on chromosome 16-and activated early in colorectal cancer ${ }^{19}$; it was shown to indirectly affect oncogenic proteins via some microRNAs in lung cancers ${ }^{20,21}$. The expression of CRNDE in EGFR-TKI-resistant cells was higher than that measured in parental EGFR-TKI-sensitive cells. Decreased expression of elF4A3, regulated by PDCD4 activation, was observed in these EGFR-TKI-resistant cells. Furthermore, the levels of MUC1 and p-EGFR were diminished in these resistant cells. CRNDE downregulates elF4A to express MUC1 and p-EGFR regardless of the upstream status of elF4A.

elF4A3 is an RNA-binding protein and a core component of the exon junction complex ${ }^{22}$, which plays important roles in mRNA splicing, transport, translation, and RNA decay ${ }^{23}$. Particularly, it is considered that elF4A3 is essential for nonsense-mediated decay, because its knockdown induced a defect in nonsense-mediated decay ${ }^{24}$. A recent study suggested that overexpression of elF4A3 was related to poor prognosis, while its dysfunction promoted tumor cell migration, invasion, and drug resistance ${ }^{25}$. Some studies reported that elF4A3 binds to IncRNAs and is involved in the development of cancer ${ }^{26,27}$.

Overexpression of MUC1-C in cancer cells was also repressed by inhibiting the elF4A RNA helicase activity. MUC1-C is a large and heavily glycosylated transmembrane protein that lubricates and protects 
the cell surface and increases cell signaling via EGFR ${ }^{28,29}$. CRNDE significantly promotes cell proliferation by mediating multiple signaling pathways and various target genes, including the PI3K/AKT signaling pathway ${ }^{19}$. Furthermore, knockdown of CRNDE recovered sensitivity to EGFR-TKI and exerted a synergistic apoptotic effect. Inhibition of CRNDE induced the activation of the elF4A/MUC1/EGFR pathway and may activate EGFR signaling (Fig. 5). Hence, CRNDE may be a promising therapeutic target in patients with EGFR-TKI-resistant EGFR-mutant NSCLC.

In summary, CRNDE induced resistance to EGFR-TKI via downregulation of elF4A-MUC1 signal transduction and may be a molecular target for overcoming resistance to EGFR-TKI. Further studies will be performed to confirm the expression of CRNDE in post-progression clinical samples after treatment with EGFR-TKI. Inhibition of CRNDE could be a promising novel therapeutic strategy for patients with EGFR-mutant NSCLC to conquer the resistance to EGFR-TKI.

\section{Materials And Methods}

\section{Cell cultures}

Four human lung adenocarcinoma cell lines were used in this study. PC-9 with an exon 19 in-frame deletion was provided from Immuno-Biological Laboratories (Gunma, Japan). NCl-HCC827 (HCC827) with a deletion in exon 19, NCl-H1975 (L858R/T790M), and H1650 with a deletion in exon 19 and PTEN null were purchased from the American Type Culture Collection (Manassas, VA, USA). These cell lines were cultured in RPMI1640 (Wako Pure Chemical Industries, Osaka, Japan) including 10\% fetal bovine serum (BioWest, Nuaillé, France), and 1\% penicillin and streptomycin (Wako Pure Chemical Industries) at $37^{\circ} \mathrm{C}$ in a $5 \% \mathrm{CO}_{2}$ incubator as previously described ${ }^{12}$. All cells were constantly examined for the absence of mycoplasma, and each experiment was performed independently three times for each condition.

\section{Drugs and cell viability assay}

Afatinib and osimertinib were obtained from Selleck Chemicals (Houston, TX, USA). We used the MTS assay to evaluate the sensitivity of the human lung adenocarcinoma cell lines to afatinib and osimertinib as previously described ${ }^{12}$. For the MTS assay, 5,000 cells per well were seeded in 96 -well tissue culture plates and incubated for $24 \mathrm{~h}$. Subsequently, the cells were treated with various concentrations of EGFRTKls or vehicle (dimethyl sulfoxide) at $37^{\circ} \mathrm{C}$ for $72 \mathrm{~h}$. Viability experiments were performed using the Cell Counting Kit 8 (Promega Corporation, Madison, WI, USA) and a microplate reader (Infinite M200 PRO; Tecan Group Ltd., Männedorf, Switzerland) according to the instructions provided by the manufacturer. The $\mathrm{IC}_{50}$ value was defined as the concentration of drug required for $50 \%$ inhibition of growth. The corrected absorbance of each sample was calculated by comparing with that of the untreated control. Each experiment was carried out thrice.

\section{Western blotting analysis}


Protein extraction, two-dimensional polyacrylamide gel electrophoresis, and transfer to nitrocellulose membrane were carried out as previously described 11,12,15. The membrane was incubated with the following antibodies: cleaved CASP3, cleaved PARP, PARP, p-EGFR, EGFR, p-AKT, AKT, p-ERK, ERK, pSTAT3, STAT3, elF4A3, p-4E-BP1, mTOR, p-P70-S6, MUC1, and MUC1-C purchased from Cell Signaling Technology (Danvers, MA, USA). The antibody to glyceraldehyde-3-phosphate dehydrogenase was purchased from Santa Cruz Biotechnology (Santa Cruz, CA, USA).

\section{RNA extraction and microarray analysis}

Total RNA from cells was extracted by TRIzol Reagent (Thermo Fisher Scientific, Waltham, MA, USA) as previously described ${ }^{10,30}$ or ISOGEN (NIPPON GENE, Tokyo, Japan).Gene expression microarray analysis was performed using a SurePrint G3 Human Gene Expression 8x60K v3 (Takara Bio, Shiga, Japan).

\section{Real-time qRT-PCR}

The cDNA of CRNDE, DGCR5, and elF4A3 was utilized for qRT-PCR analysis using the THUNDERBIRD SYBR qPCR/RT Set III (TOYOBO, Osaka, Japan) according to the instructions provided by the manufacturer. The expression of these genes was examined through the TaqMan Gene Expression Assay (Thermo Fisher Scientific) ${ }^{31,32}$. Gene expression levels were quantified using the $2^{-\Delta \Delta C t}$ method.

\section{SiRNA transfection}

The siRNA targeting CRNDE and negative control were purchased from Thermo Fisher Scientific. All siRNAs were treated with Lipofectamine RNAiMAX (Thermo Fisher Scientific) transfection reagent 24 hours after seeding, according to the instructions provided by the manufacturer. We transfected the siRNA complexes into cells at a final concentration of $50 \mathrm{nM}$. After 6 hours from the addition of the siRNA complexes, cells were seeded in RPMI 1640 containing $10 \%$ fetal bovine serum, and $1 \%$ penicillin and streptomycin and incubated at $37^{\circ} \mathrm{C}$ for 48 hours.

\section{Statistical analysis}

Differences in categorical outcomes were assessed by the chi-squared test. The statistical significance of differences decided with the standard Student's t-test. P-value of $<0.05$ was considered statistically significant. Analyses were carried out by the statistical software JMP 9 (SAS Institute, Cary, NC, USA).

\section{Bioinformatics}

The transcriptome (consisting of mRNAs and IncRNAs) was used as a reference for mapping. LncRNA sequencing data were extracted from LNCipedia ${ }^{33}$ (https://Incipedia.org/downloads/Incipedia_5_0.fasta), including Refseq, Ensembl, Gencode, Broad Institute (Human Body Map lincRNAs), NONCODE and FANTOM CAT to establish a IncRNA database. The mRNA sequencing data were extracted from Gencode $^{34}$ (ftp://ftp.ebi.ac.uk/pub/databases/gencode/Gencode_human/release_28/gencode.v28.transcripts.fa.gz) 
to establish a mRNA database. The sequencing data were extracted from the probe information site of Agilent Inc. The candidate RNA, with more than two-fold change in levels between parental cells and resistant sublines, was detected. The protein linked to the candidate RNA was detected using RNA-Protein binding data (http://starbase.sysu.edu.cn/starbase2/index.php) ${ }^{35}$.

\section{Declarations}

\section{Author Contributions}

ST, RN, and MS wrote and revised the manuscript and prepared all figures and tables. All authors reviewed the manuscript.

\section{Acknowledgements}

We thank Ms. Chiba Mika of Nippon Medical School (Tokyo, Japan) for her excellent technical assistance. This study was supported in part by a grant-in-aid from the Ministry of Education, Culture, Sports, Science, and Technology of Japan (grant 16K09592 to MS) and a Clinical Rebiopsy Bank Project for Comprehensive Cancer Therapy Development (to AG and MS).

\section{Competing Interests}

MS has received speakers' bureau honoraria from AstraZeneca Co., Ltd. and Boehringer Ingelheim Pharmaceuticals, Inc. AG has received a commercial research grant and speakers' bureau honoraria from AstraZeneca Co. Ltd. and Boehringer Ingelheim Pharmaceuticals, Inc. The other authors have no conflicts of interest to declare.

\section{Data Availability}

All data generated or analyzed during this study are included in this published article and its Supplementary Information files. Microarray data have been deposited in the NCBI Gene Expression Omnibus (GEO; http://www.ncbi.nlm.nih.gov/geo/) and are accessible through the GEO series accession number GSE163913.

\section{References}

1. Maemondo, M. et al. Gefitinib or chemotherapy for non-small-cell lung cancer with mutated EGFR. The New England journal of medicine. 362, 2380-2388 https://doi.org/10.1056/NEJMoa0909530 (2010).

2. Mitsudomi, T. et al. Gefitinib versus cisplatin plus docetaxel in patients with non-small-cell lung cancer harbouring mutations of the epidermal growth factor receptor (WJTOG3405): an open label, randomised phase 3 trial. Lancet Oncol. 11, 121-128 https://doi.org/10.1016/s14702045(09)70364-x (2010). 
3. Zhou, C. et al. Erlotinib versus chemotherapy as first-line treatment for patients with advanced EGFR mutation-positive non-small-cell lung cancer (OPTIMAL, CTONG-0802): a multicentre, open-label, randomised, phase 3 study. Lancet Oncol. 12, 735-742 (2011).

4. Rosell, R. et al. Erlotinib versus standard chemotherapy as first-line treatment for European patients with advanced EGFR mutation-positive non-small-cell lung cancer (EURTAC): a multicentre, openlabel, randomised phase 3 trial. Lancet Oncol. 13, 239-246 (2012).

5. Sequist, L. V. et al. Phase III study of afatinib or cisplatin plus pemetrexed in patients with metastatic lung adenocarcinoma with EGFR mutations. Journal of clinical oncology: official journal of the American Society of Clinical Oncology. 31, 3327-3334 https://doi.org/10.1200/jco.2012.44.2806 (2013).

6. Wu, Y. L. et al. Afatinib versus cisplatin plus gemcitabine for first-line treatment of Asian patients with advanced non-small-cell lung cancer harbouring EGFR mutations (LUX-Lung 6): an open-label, randomised phase 3 trial. Lancet Oncol. 15, 213-222 https://doi.org/10.1016/s14702045(13)70604-1 (2014).

7. Mok, T. S. et al. Osimertinib or Platinum-Pemetrexed in EGFR T790M-Positive Lung Cancer. The New England journal of medicine. 376, 629-640 https://doi.org/10.1056/NEJMoa1612674 (2017).

8. Ramalingam, S. S. et al. Overall Survival with Osimertinib in Untreated, EGFR-Mutated Advanced NSCLC. The New England journal of medicine. 382, 41-50 https://doi.org/10.1056/NEJMoa1913662 (2020).

9. $\mathrm{Yu}, \mathrm{H}$. A. et al. Analysis of tumor specimens at the time of acquired resistance to EGFR-TKI therapy in 155 patients with EGFR-mutant lung cancers. Clinical cancer research: an official journal of the American Association for Cancer Research. 19, 2240-2247 https://doi.org/10.1158/1078-0432.ccr12-2246 (2013).

10. Seike, M. et al. MiR-21 is an EGFR-regulated anti-apoptotic factor in lung cancer in never-smokers. Proceedings of the National Academy of Sciences of the United States of America. 106, 12085$12090 \mathrm{https}: / /$ doi.org/10.1073/pnas.0905234106 (2009).

11. Kitamura, K. et al. MiR-134/487b/655 cluster regulates TGF- $\beta$-induced epithelial-mesenchymal transition and drug resistance to gefitinib by targeting MAGI2 in lung adenocarcinoma cells. Molecular cancer therapeutics. 13, 444-453 https://doi.org/10.1158/1535-7163.mct-13-0448 (2014).

12. Takahashi, A. et al. Ankyrin Repeat Domain 1 Overexpression is Associated with Common Resistance to Afatinib and Osimertinib in EGFR-mutant Lung Cancer. Scientific reports. 8, 14896 https://doi.org/10.1038/s41598-018-33190-8 (2018).

13. Cheetham, S. W., Gruhl, F., Mattick, J. S. \& Dinger, M. E. Long noncoding RNAs and the genetics of cancer. British journal of cancer. 108, 2419-2425 https://doi.org/10.1038/bjc.2013.233 (2013).

14. Chen, J., Wang, R., Zhang, K. \& Chen, L. B. Long non-coding RNAs in non-small cell lung cancer as biomarkers and therapeutic targets. Journal of cellular and molecular medicine. 18, 2425-2436 https://doi.org/10.1111/jcmm.12431 (2014). 
15. Sugano, T. et al. Inhibition of ABCB1 Overcomes Cancer Stem Cell-like Properties and Acquired Resistance to MET Inhibitors in Non-Small Cell Lung Cancer. Molecular cancer therapeutics. 14, 2433-2440 https://doi.org/10.1158/1535-7163.mct-15-0050 (2015).

16. Iwakiri, J., Hamada, M. \& Asai, K. Bioinformatics tools for IncRNA research. Biochimica et biophysica acta. 1859, 23-30 https://doi.org/10.1016/j.bbagrm.2015.07.014 (2016).

17. Chen, E. G., Zhang, J. S., Xu, S., Zhu, X. J. \& Hu, H. H. Long non-coding RNA DGCR5 is involved in the regulation of proliferation, migration and invasion of lung cancer by targeting miR-1180. American journal of cancer research. 7, 1463-1475 (2017).

18. Wang, J., Shu, H. Z., Xu, C. Y. \& Guo, S. G. LncRNA DGCR5 promotes non-small cell lung cancer progression via sponging miR-218-5p. European review for medical and pharmacological sciences. 23, 9947-9954 https://doi.org/10.26355/eurrev_201911_19561 (2019).

19. Graham, L. D. et al. Colorectal Neoplasia Differentially Expressed (CRNDE), a Novel Gene with Elevated Expression in Colorectal Adenomas and Adenocarcinomas. Genes \& cancer. 2, 829-840 https://doi.org/10.1177/1947601911431081 (2011).

20. Fan, Y. F., Yu, Z. P. \& Cui, X. Y. IncRNA Colorectal Neoplasia Differentially Expressed (CRNDE) Promotes Proliferation and Inhibits Apoptosis in Non-Small Cell Lung Cancer Cells by Regulating the miR-641/CDK6 Axis. Medical science monitor: international medical journal of experimental and clinical research. 25, 2745-2755 https://doi.org/10.12659/msm.913420 (2019).

21. Jing, H., Xia, H., Qian, M. \& Lv, X. Long noncoding RNA CRNDE promotes non-small cell lung cancer progression via sponging microRNA-338-3p. Biomedicine \& pharmacotherapy $=$ Biomedecine \& pharmacotherapie. 110, 825-833 https://doi.org/10.1016/j.biopha.2018.12.024 (2019).

22. Saulière, J. et al. CLIP-seq of elF4AIll reveals transcriptome-wide mapping of the human exon junction complex. Nature structural \& molecular biology. 19, 1124-1131 https://doi.org/10.1038/nsmb.2420 (2012).

23. Rocak, S. \& Linder, P. DEAD-box proteins: the driving forces behind RNA metabolism. Nature reviews. Molecular cell biology. 5, 232-241 https://doi.org/10.1038/nrm1335 (2004).

24. Shibuya, T., Tange, T., Sonenberg, N. \& Moore, M. J. elF4All binds spliced mRNA in the exon junction complex and is essential for nonsense-mediated decay. Nature structural \& molecular biology. 11, 346-351 https://doi.org/10.1038/nsmb750 (2004).

25. Lin, Y. et al. Systematic Analysis of Gene Expression Alteration and Co-Expression Network of Eukaryotic Initiation Factor 4A-3 in Cancer. Journal of Cancer. 9, 4568-4577 https://doi.org/10.7150/jca.27655 (2018).

26. Li, N. \& Zhan, X. Anti-parasite drug ivermectin can suppress ovarian cancer by regulating IncRNAEIF4A3-mRNA axes. The EPMA journal. 11, 289-309 https://doi.org/10.1007/s13167-020-00209-y (2020).

27. Han, D. et al. Long noncoding RNA H19 indicates a poor prognosis of colorectal cancer and promotes tumor growth by recruiting and binding to elF4A3. Oncotarget. 7, 22159-22173 https://doi.org/10.18632/oncotarget.8063 (2016). 
28. Jin, C., Rajabi, H., Rodrigo, C. M., Porco, J. A. Jr. \& Kufe, D. Targeting the elF4A RNA helicase blocks translation of the MUC1-C oncoprotein. Oncogene. 32, 2179-2188 https://doi.org/10.1038/onc.2012.236 (2013).

29. Engel, B. J., Bowser, J. L., Broaddus, R. R. \& Carson, D. D. MUC1 stimulates EGFR expression and function in endometrial cancer. Oncotarget. 7, 32796-32809 https://doi.org/10.18632/oncotarget.8743 (2016).

30. Seike, M. et al. Use of a cytokine gene expression signature in lung adenocarcinoma and the surrounding tissue as a prognostic classifier. Journal of the National Cancer Institute. 99, 12571269 https://doi.org/10.1093/jnci/djm083 (2007).

31. Bustin, S. A. Absolute quantification of mRNA using real-time reverse transcription polymerase chain reaction assays. Journal of molecular endocrinology. 25, 169-193 https://doi.org/10.1677/jme.0.0250169 (2000).

32. Ji, P. et al. MALAT-1, a novel noncoding RNA, and thymosin beta4 predict metastasis and survival in early-stage non-small cell lung cancer. Oncogene. 22, 8031-8041 https://doi.org/10.1038/sj.onc.1206928 (2003).

33. Volders, P. J. et al. LNCipedia 5: towards a reference set of human long non-coding RNAs. Nucleic acids research. 47, D135-D139 https://doi.org/10.1093/nar/gky1031 (2019).

34. Frankish, A. et al. GENCODE reference annotation for the human and mouse genomes. Nucleic acids research. 47, D766-D773 https://doi.org/10.1093/nar/gky955 (2019).

35. Li, J. H., Liu, S., Zhou, H., Qu, L. H. \& Yang, J. H. starBase v2.0: decoding miRNA-ceRNA, miRNAncRNA and protein-RNA interaction networks from large-scale CLIP-Seq data. Nucleic acids research. 42, D92-97 https://doi.org/10.1093/nar/gkt1248 (2014).

\section{Figures}


a
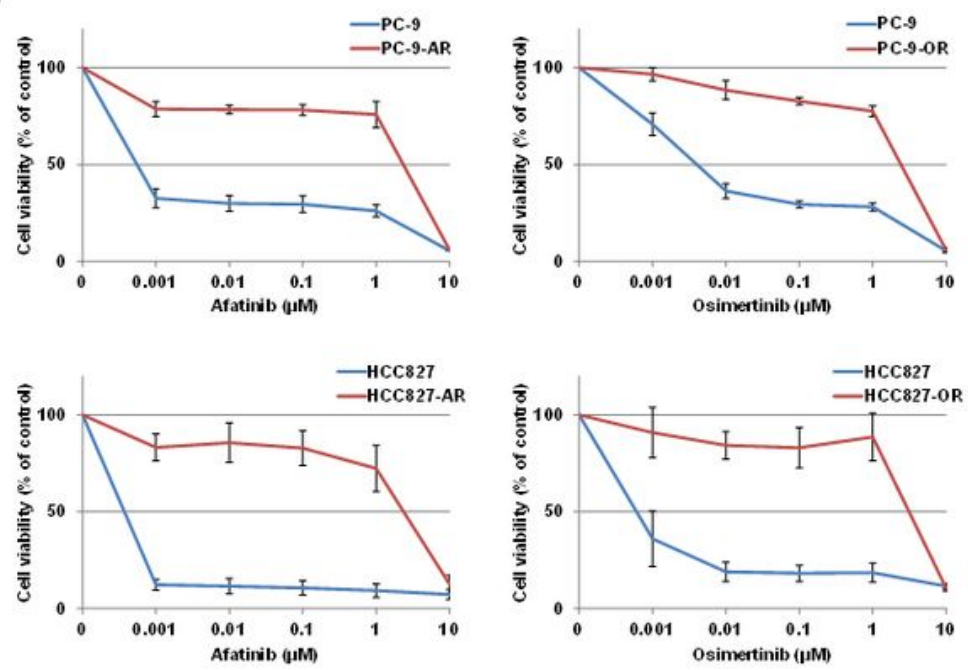

b

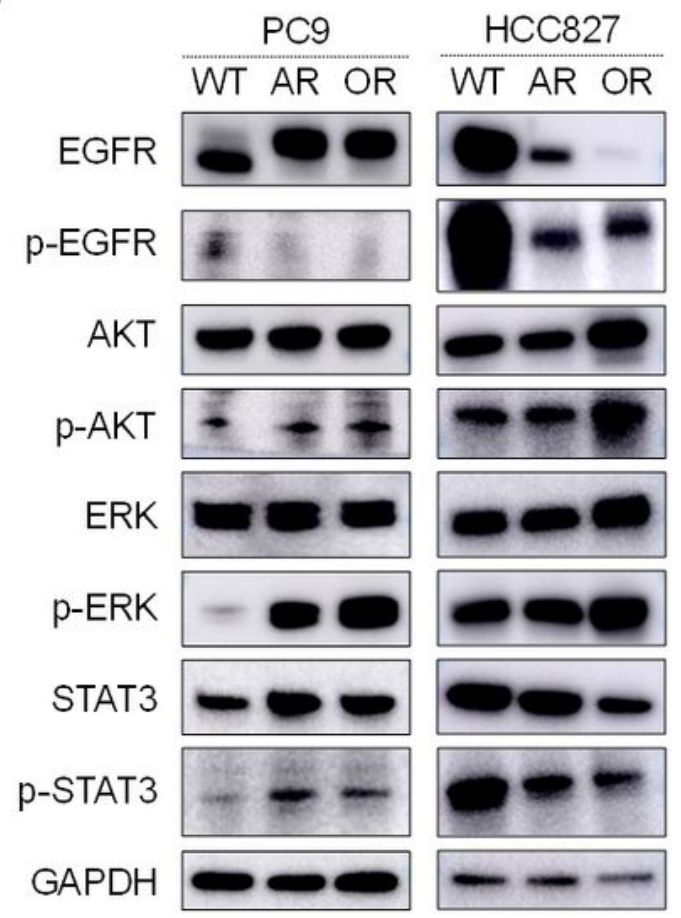

\section{Figure 1}

Establishment of EGFR-TKI-resistant lung cancer cell lines. (a) Establishment of afatinib-resistant (AR) and osimertinib-resistant (OR) PC-9 and HCC-827 cell lines. PC-9-afatinib-resistant cell line (PC-9-AR); PC9-osimertinib-resistant cell line (PC-9-OR); HCC827-afatinib-resistant cell line (HCC827-AR); and HCC827osimertinib-resistant cell line (HCC827-OR). The results of cell viability assays are shown. (b) Protein expression levels of EGFR signaling pathway molecules, namely AKT, ERK, and STAT3. EGFR-TKI, 
epidermal growth factor receptor-tyrosine kinase inhibitor; ERK, extracellular signal-regulated kinase; GAPDH, glyceraldehyde-3-phosphate dehydrogenase; WT, wild type

a
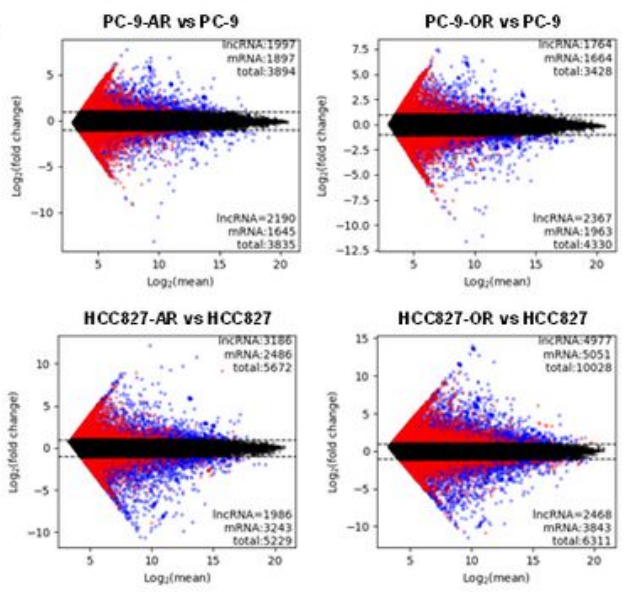

b
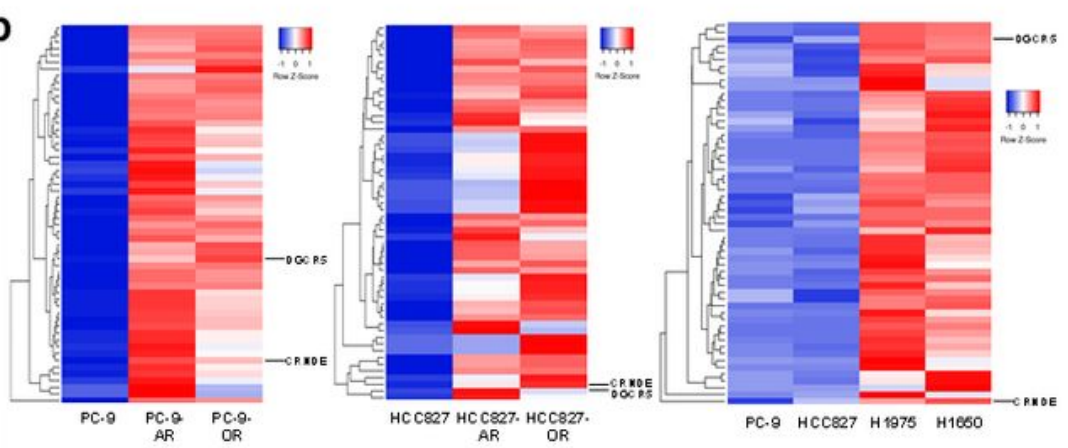

C
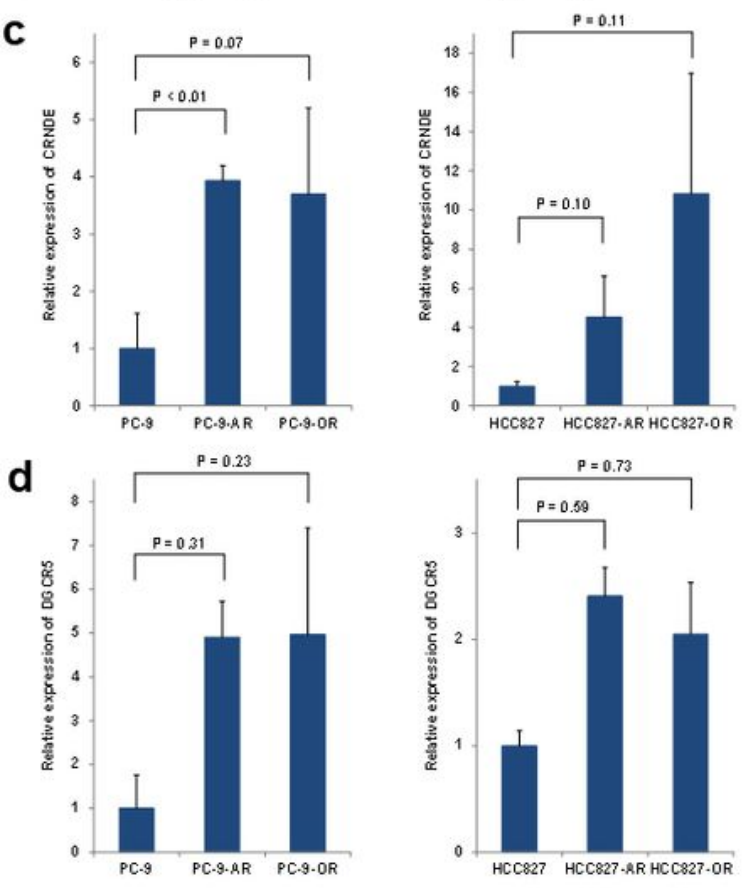

Figure 2

Overexpression of CRNDE and DGCR5 in EGFR-TKIs-resistant NSCLC cells. (a) Microarray analyses: The candidate IncRNAs (blue color) and mRNAs (Red color) were determined by differences in expression using a cut-off value of more that two-fold change between resistant and parental cells. (b) The 
expression of CRNDE and DGCR5 IncRNAs was higher in all examined resistant cell lines than in the parental cell lines. In addition, these IncRNAs showed higher expression levels in intrinsic resistant cells (i.e., H1975 and H1650) than in sensitive cells (i.e., PC9 and HCC827). (c) The expression of CRNDE was higher in all examined resistant cell lines than in the parental cell lines. (d) The expression of DGCR5 was higher in all examined resistant cell lines than in the parental cell lines. CRNDE, colorectal neoplasia differentially expressed; DGCR5, DiGeorge syndrome critical region gene 5; EGFR-TKI, epidermal growth factor receptor-tyrosine kinase inhibitor; IncRNA, long non-coding RNA; mRNA, messenger RNA; NSCLC, non-small cell lung cancer 
a

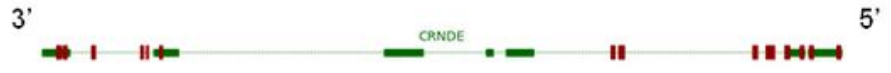

b
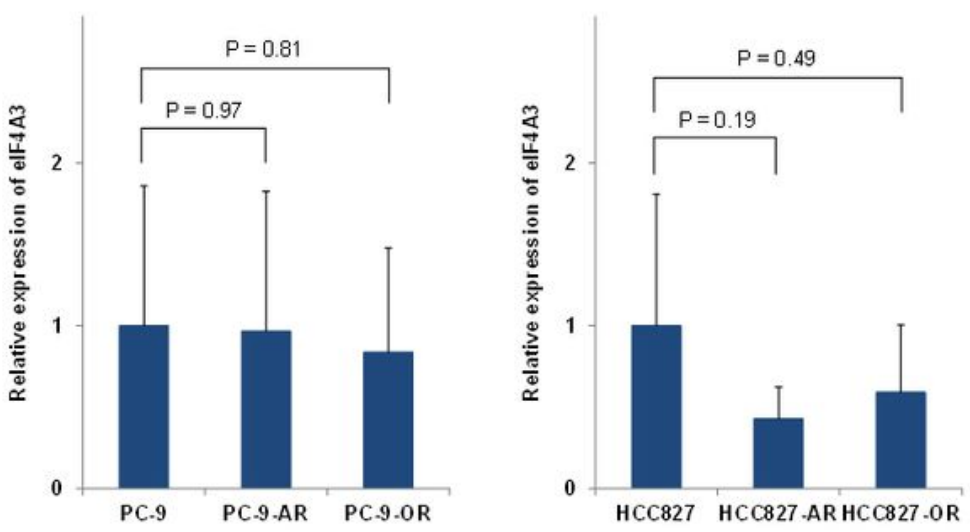

C

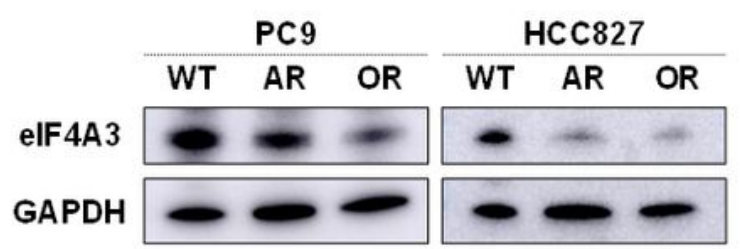

d

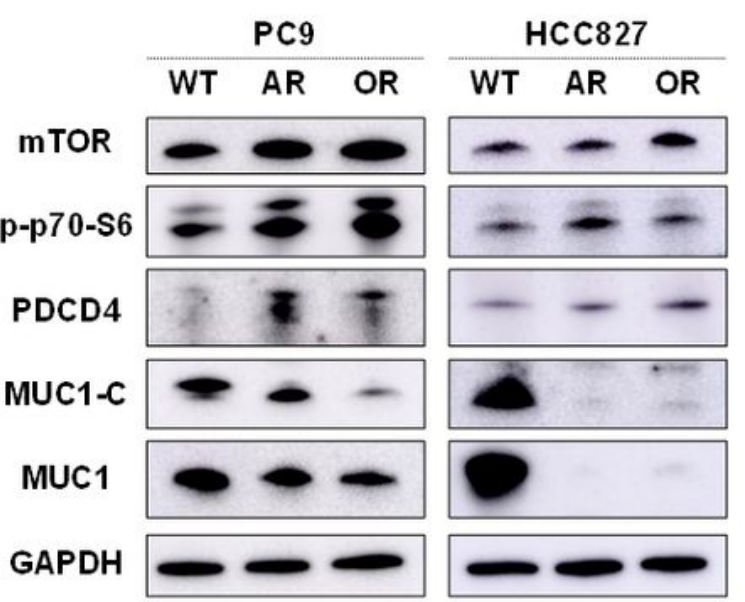

Figure 3

CRNDE decreased the levels of elF4A3 downstream molecules by binding elF4A3 in EGFR-TKI-resistant NSCLC cells (a) A motif of the elF4A3-binding site on CRNDE. elF4A3-binding sites are shown as red rectangles. (b) qRT-PCR revealed the RNA levels of elF4A3 expression. The expression of elF4A3 may decline in the established EGFR-TKI-resistant cells. (c) Western blotting analysis showed that elF4A3 expression was declined in the EGFR-TKI-resistant cells. (d) Western blotting analysis demonstrated 
increased protein expression of the AKT/mTOR signaling molecules and decreased protein expression of the elF4A downstream signaling molecules in EGFR-TKI-resistant cells. AR, afatinib-resistant; CRNDE, colorectal neoplasia differentially expressed; elF4A3, eukaryotic translation initiation factor 4A3; EGFRTKI, epidermal growth factor receptor-tyrosine kinase inhibitor; GAPDH, glyceraldehyde-3-phosphate dehydrogenase; mTOR, mechanistic target of rapamycin kinase; MUC1, mucin 1; NSCLC, non-small cell lung cancer; OR, osimertinib-resistant; PDCD4, programmed cell death 4; qRT-PCR, quantitative reverse transcription-polymerase chain reaction; WT, wild type
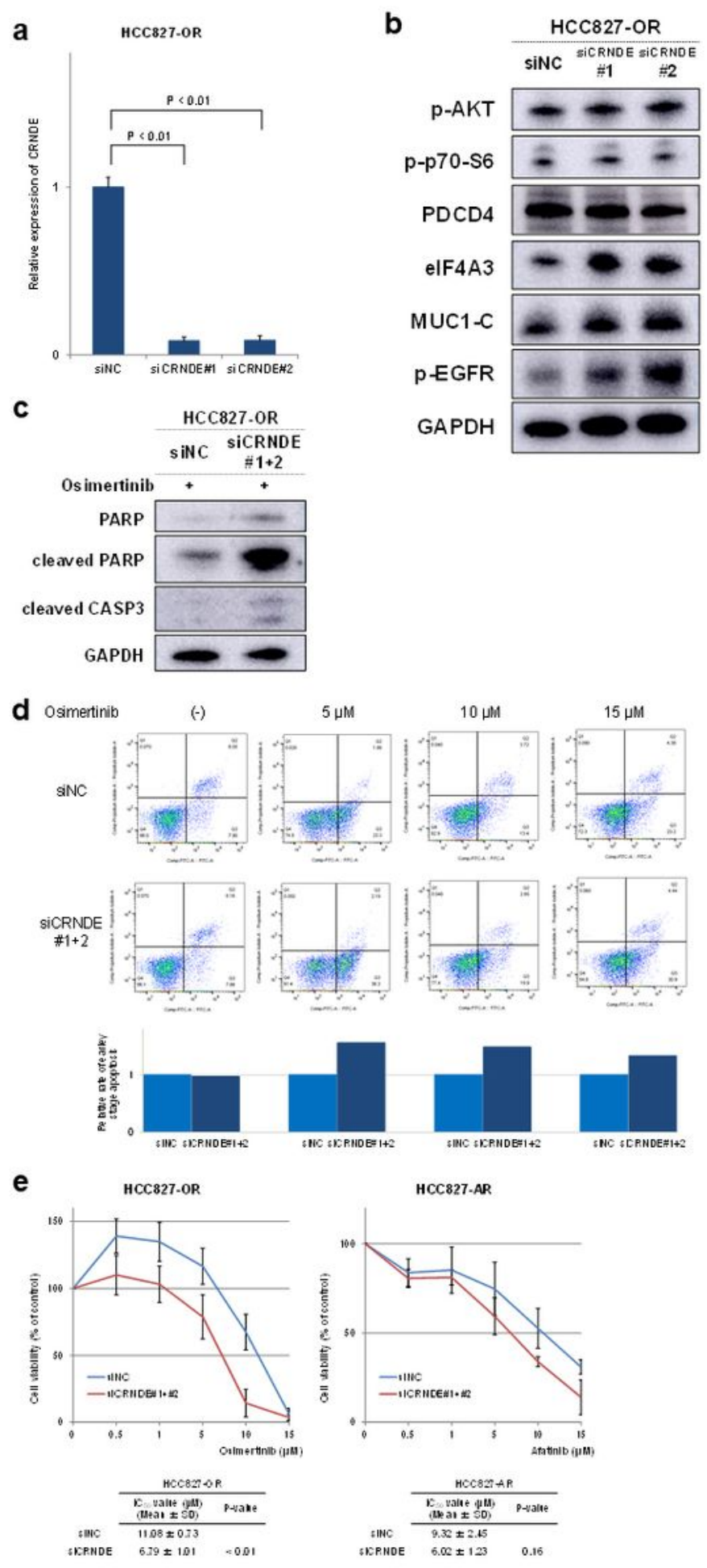


\section{Figure 4}

Knockdown of CRNDE induced apoptosis and recovered sensitivity to EGFR-TKIs in drug-resistant cells (a) qRT-PCR showed that transfection of HCC827-OR cells with siRNA for CRNDE inhibited its expression. Fold change for the decrease in CRNDE relative expression. (b) Western blotting analysis showed constant protein expression of the AKT/mTOR signaling molecules and increased protein expression of the elF4A3 downstream signaling molecules after knockdown of CRNDE in HCC827-OR cells. (c) Western blotting analysis showed overexpression of apoptosis-related proteins after treatment with osimertinib following knockdown of CRNDE in HCC827-OR cells. (d) Apoptosis induced by osimertinib at some concentrations after knockdown of CRNDE in HCC827-OR cells, as determined by annexin V-propidium iodide (PI) staining. (e) Dose-dependent sensitivity of EGFR-TKI-resistant cell lines to afatinib and osimertinib after knockdown of CRNDE by siRNA. AR, afatinib-resistant; CASP3, caspase 3; CRNDE, colorectal neoplasia differentially expressed; EGFR-TKI, epidermal growth factor receptor-tyrosine kinase inhibitor; elF4A3, eukaryotic translation initiation factor 4A3; GAPDH, glyceraldehyde-3-phosphate dehydrogenase; mTOR, mechanistic target of rapamycin kinase; MUC1, mucin 1; OR, osimertinib-resistant; PARP, poly(ADP-ribose) polymerase; PDCD4, programmed cell death 4; qRT-PCR, quantitative reverse transcription-polymerase chain reaction; SD, standard deviation; siRNA, small-interfering RNA 


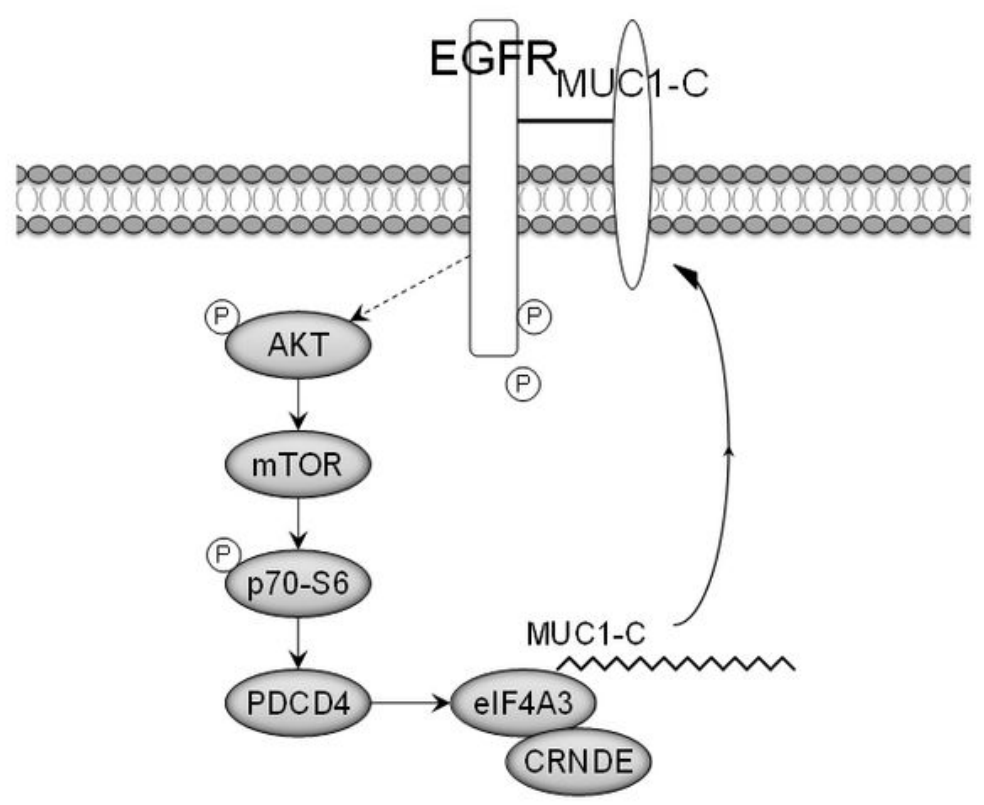

$\mathrm{CRNDE} \downarrow \Rightarrow \mathrm{eIF} 4 \mathrm{~A} \uparrow \Rightarrow \mathrm{MUC} 1 \uparrow \Rightarrow \mathrm{EGFR} \uparrow$

\section{Figure 5}

Schematic results. In EGFR-TKI-resistant cells, the expression levels of elF4A3 and its downstream signaling molecules (i.e., MUC1 and p-EGFR) were decreased, whereas those of its upstream molecules (i.e., AKT, mTOR, p70-S6, and PDCD4) were increased. Following inhibition of CRNDE, the expression levels of upstream molecules AKT, mTOR, and p70-S6 were not changed. In contrast, EIF4A/MUC1/pEGFR expression was increased. CRNDE, colorectal neoplasia differentially expressed; EGFR-TKI, 
epidermal growth factor receptor-tyrosine kinase inhibitor; elF4A3, eukaryotic translation initiation factor 4A3; mTOR, mechanistic target of rapamycin kinase; MUC1, mucin 1; PDCD4, programmed cell death 4

\section{Supplementary Files}

This is a list of supplementary files associated with this preprint. Click to download.

- SupplementaryFigureS1.tif

- SupplementaryFigureS2originalpicturesofFig1b.tif

- SupplementaryFigureS3originalpicturesofFig3c.tif

- SupplementaryFigureS4originalpicturesofFig3d.tif

- SupplementaryFigureS5originalpicturesofFig4b.tif

- SupplementaryFigureS6originalpicturesofFig4c.tif

- SupplementaryTableS1.docx 